Michael, K.D., Furr, R.M., Masters, K.S., Collett, B.R., Spielmans, G.I., Ritter, K., Veeder, M.A., Treiber, K., \& Cullum, J.L. (2009). Using the MMPI-2 to predict symptom reduction during psychotherapy in a sample of community outpatients. Journal of Contemporary Psychotherapy, 39:3, pp. 157-163. (ISSN: 0022-0116) Springer Verlag DOI: 10.1007/s10879008-9109-x

Keywords: Psychotherapy | Outcome | MMPI-2 | OQ-45

\title{
Using the MMPI-2 to Predict Symptom Reduction during Psychotherapy in a Sample of Community Outpatients
}

\author{
Kurt D. Michael, R. Michael Furr, Kevin S. Masters, Brent R. Collett, Glen I. \\ Spielmans, Kathrin Ritter, Marietta A. Veeder, Katherine Treiber and Jodi L. \\ Cullum
}

\begin{abstract}
In the present study, potential MMPI-2 predictors of psychotherapy outcome were examined in a community clinical sample of 51 patients seeking treatment at a university training clinic. Results indicated that particular MMPI-2 scales (L, F, Pd, Pa, Sc, Trt) were the most predictive of initial levels of patient distress, whereas three other clinical scales (Hs, D, Hy) were significantly associated with actual symptom reduction over time. The clinical implications of these data include the use of the MMPI-2 in clinical practice as a means to frame the provision of direct feedback to patients regarding the likelihood of treatment response, which in turn, might actually have therapeutic benefits. The limitations of the study are reviewed and suggestions for future research are offered, including the potential use of widely known and utilized instruments in helping to predict response to psychotherapy.
\end{abstract}

\section{ARTICLE}

\section{Introduction}

Historically, the evaluation of whether psychotherapy patients benefited from treatment was a frequent topic of empirical inquiry and debate (e.g., Eysenck 1952; Smith et al. 1980). However, since then, a substantial body of literature has emerged in support of psychotherapy overall (Lambert et al.2003). Perhaps tantamount to the issue of whether someone improves during psychotherapy is the question of who is likely to benefit from the psychotherapeutic endeavor. That is, what is the relationship between client variables and psychotherapy outcome, including demographic features, severity of symptoms, and personality variables? With respect to personality variables, researchers have long sought to identify predictors of psychotherapy outcome. Indeed, measures of personality, such as the Minnesota Multiphasic Personality Inventory (MMPI; Hathaway and McKinley 1983) have received a great deal of attention in the 
literature. However, the results using the MMPI and other measures of personality (e.g., Rorschach) as predictors of psychotherapy outcome have been equivocal (Garfield 1994). Moreover, although many practitioners are quite familiar with the MMPI and many still use it regularly (Hill and Lambert 2003), its use in predicting and/or evaluating outcome has been complicated by the time and energy required to administer, score and interpret the measure in addition to structural and psychometric concerns (see Helmes and Reddon 1993 for a complete review).

Nonetheless, since the revision of the MMPI (MMPI-2; Butcher et al. 2001), there has been renewed interest in using this revised and enhanced instrument as a predictor of treatment outcome, therefore increasing its utility in everyday clinical practice beyond an evaluation and/or diagnostic tool (Graham 2005). The MMPI-2 has been used to predict outcome for a variety of conditions, including but not limited to: posttraumatic stress disorder (Forbes et al. 2002), chronic back pain (e.g., Vendrig et al. 2000), substance abuse disorders (e.g., Gilmore et al. 2001), and even surgical treatment of lumbar pain (Masters et al.2003). The samples utilized in such studies range from hospitalized patients and those with specific diagnostic presentations to outpatient samples of general psychotherapy patients with a broad mixture of presenting problems. Whereas some studies have supported the predictive power of the MMPI-2 for psychotherapy outcome (e.g., Forbes et al. 2002) other studies have failed to find evidence of incremental validity when using the instrument to predict outcome (e.g., Lima et al. 2005; Minnix et al. 2005).

Moreover, the MMPI-2 variables that have previously been studied range from the basic validity and clinical scales to numerous content and supplementary scales. Unfortunately, there is tremendous variability in the specific MMPI-2 scales utilized as predictors of outcome across studies and many of the investigations have not been replicated (Garfield 1994). Complicating matters is the fact that the definition of outcome across most studies varies widely, ranging from the level of posttreatment symptom severity (Forbes et al. 2002) in one study to whether the patient terminated from therapy prematurely in another (Hilsenroth et al. 1995).

Despite the aforementioned limitations in the current literature, some specific MMPI-2 scales that have been found to be relatively powerful predictors of outcome. Forbes et al. (2002) used the MMPI-2 as a predictor of symptom change in a sample of 141 Vietnam veterans with chronic posttraumatic stress disorder (PTSD). The authors reported that although initial PTSD symptom severity accounted for the most variance (i.e., 23\%) among the predictors of outcome, there was a statistically significant inverse relationship between the Psychopathic Deviate (Pd) scale and PTSD symptom change at the end of treatment (i.e., additional 3.8\% of the variance). That is, elevated scores on Pd at pretreatment were associated with smaller changes in PTSD symptoms (i.e., higher PTSD symptoms) at posttreatment. These data are generally consistent with the MMPI-2 interpretive guidelines as described by Graham (2005), wherein elevations on scales such as Pd may not bode well for psychotherapy outcomes. Graham (2005) noted "high scorers often are perceived as good candidates for psychotherapy. Unfortunately, the prognosis for 
change is poor. Although they may agree to treatment to avoid something more unpleasant (e.g., jail, divorce), they generally are unable to accept blame for their own problems, and they terminate treatment as soon as possible" (p. 75). However, Chisholm et al. (1997) reported significant positive associations between the Pd and Pt (Psychasthenia) scales with improvements in global psychopathology (measured by therapist rating) and improvements in current functioning (therapist rating), respectively.

So, it appears that the relationships between MMPI-2 scales and outcome are not necessarily consistent. In an effort to address these equivocal findings and to expand our understanding of how MMPI-2 scales might be helpful in predicting outcome, some authors suggested moving beyond the basic validity and clinical scales to the various content and supplementary scales. Graham (2005) asserted that "in most cases relationships between content scales and extratest characteristics are stronger than between clinical scales and those characteristics" (p. 139). Some of the most commonly studied scales (other than validity and clinical scales) include the Depression (DEP), Anxiety (ANX), Negative Treatment Indicator (TRT), and Ego Strength (Es) scales. Chisholm et al. (1997) reported that DEP, ANX, and TRT were correlated with various indices of outcome and all were generally superior to the basic clinical scales in predicting the effects of psychotherapy. However, with respect to the TRT in particular, the findings have been equivocal despite the fact that the scale purportedly measures the presence of "negative treatment attitudes" that might hinder a successful therapeutic outcome (Graham 2005). Clark (1996) reported that elevations on the TRT scale were associated with both positive and negative treatment outcomes for men with chronic pain, depending on the measures used. Thus, the findings regarding the TRT scale were actually contradictory in the same study! Further, Minnix et al. (2005) as well as Craig and Olson (2004) failed to find any support for the TRT as a predictor of treatment outcome.

With respect to the Es scale, Barron (1953) reportedly predicted outcome in a sample of 33 patients who underwent psychotherapy for 6 months. However, these findings were not replicated after several attempts over the next two decades (e.g., Getter and Sundland 1962). More recent attempts to substantiate Barron's initial findings regarding the Es scale have been mixed. For example, while Luborsky et al. (1980) failed to support the Es scale's utility in predicting treatment outcome, Shepherd (1997) reported that psychotherapy clients with higher pretreatment Es scores were assessed to be more improved than those patients with lower Es scores at posttreatment.

In summary, it appears that several of the MMPI-2 scales have some utility in predicting therapy outcome for a variety of conditions, but many of the findings are equivocal. Moreover, in addition to predicting initial levels of distress, it is important to clarify how the MMPI-2 scales are associated with the psychotherapy process over time. Given this state of the literature, the primary purpose of this study was to assess the utility of particular MMPI-2 scales in predicting who will evidence the most symptom reduction in a community sample of outpatients referred for psychotherapy after taking into account, initial symptom severity. In addition to the clinical 
scales, we also used three content scales (ANX, DEP, TRT) and the Es supplementary scale as potential predictors of outcome given the reported findings that suggest they might be better predictors beyond what the clinical scales provide (e.g., Barron 1953; Chisholm et al. 1997; Graham 2005) and in some cases where the scale (i.e., TRT) was designed to help to identify those less amenable to treatment (Butcher et al. 1990).

\section{Method}

\section{Participants}

Participants were 51 patients (53\% female) drawn from a community university-based clinic. The mean age of the participants was $27.16(\mathrm{SD}=9.36)$ and the cultural background was predominantly Caucasian (92\%), followed by Latino (5\%), Asian (1\%), African-American (1\%), and American Indian (1\%). Upon referral, patients completed a diagnostic evaluation including a clinical interview, the MMPI-2, and the OQ-45. Each of the patient MMPI-2 profiles were valid for interpretive purposes and each of the 51 patients presented with an elevated OQ-45 total score (i.e., $\geq 63$ ). This score has been established as an appropriate cutoff in distinguishing between clinical and non-clinical levels of overall distress on the OQ-45 (Lambert et al. 2004, see below). Of the 51 patients, 37 (72.5\%) were given a primary Axis I or II DSM-IV-TR (American Psychiatric Association 2000) diagnosis, the most common of which was a mood disorder $(n=20)$ followed by an anxiety disorder $(n=8)$. The mean BDI score (only administered at intake) for the 51 patients was $20.01(\mathrm{SD}=10.83)$. The remaining $14(27.5 \%)$ patients received treatment for presenting problems and V-codes (e.g., 5 patients with Partner Relational Problem) or elevated symptoms of depression as measured by the BDI $(M=19.41$; $\mathrm{SD}=8.43$ ), but who did not have a documented Axis I or II DSM diagnosis at intake.

Therapists were all doctoral candidates who had completed at least 1 year of graduate coursework and clinical training. There were 23 therapists (12 female, 11 male) who provided the psychotherapy. In conjunction with clinical practica, doctoral candidates received $1 \mathrm{~h}$ of weekly individual supervision and $3 \mathrm{~h}$ of weekly group supervision from one of five clinical faculty members (three women, two men), all of whom were licensed clinical psychologists (range of clinical experience: 4-25 years; 4 of 5 with $>10$ years experience). The cultural background of the supervisors was predominantly Caucasian $(\mathrm{N}=4)$ with one being American Indian. The theoretical orientation among the doctoral trainees and supervisors was largely cognitive-behavioral. As is clinic policy for all patients, each of the evaluation and treatment sessions were videotaped for review in supervision. 


\section{Measures \\ OQ-45}

The Outcome Questionnaire-45 (OQ-45; Lambert et al.1996a) is a 45-item multiple-choice selfreport instrument that assesses client symptoms during active treatment and follow-up phases. It is used to evaluate client symptoms in three areas of psychological functioning: (1) subjective discomfort (intrapsychic functioning), (2) interpersonal functioning, and (3) social role performance. Previous studies indicate that the most reliable index of treatment outcome is based upon the total OQ-45 score (Lambert et al. 2004; Vermeersch et al. 2000). Developed from normative data for outpatients and community non-patients $(\mathrm{N}=1,157)$, an OQ-45 total score of 63 was identified as an appropriate cutoff in distinguishing between clinical and non-clinical levels (Lambert et al. 2004).

The OQ-45 has a demonstrated reliability for undergraduate students, with a 3 week test-retest reliability of .84 and internal consistency of .93 (Lambert et al. 1996b). Convergent validity for the OQ-45 is well-supported by correlations with the Inventory of Interpersonal Problems ( $\mathrm{r}=$ $.63)$, the Beck Depression Inventory $(r=.62)$, the General Severity Index $(r=.72)$, the State Anxiety $(r=.64)$ and Trait Anxiety scales $(r=.80)$ of the State Trait Anxiety Inventory (Lambert et al.1996b). Given that we were interested in predicting change over the course of psychotherapy, it is important to note that the OQ-45 has demonstrated sensitivity to change (Vermeersch et al. 2000).

\section{MMPI-2}

The MMPI-2 (Butcher et al. 2001) is a well known and widely used psychological test consisting of 567 true-false items. It traditionally yields scores on four validity scales and 10 clinical scales, although numerous other scales may be scored. For this study, the clinical, select content (Anxiety, Depression, Negative Treatment Indicators) and supplementary (Ego Strength) scales were analyzed. Regarding the content and supplementary scales, nine of the patients had missing data and thus only 42 patients were included in these analyses. Scores on the Mf (MasculinityFemininity) scale were not included, given that this was a mixed-gender sample and sample size was insufficient to evaluate outcomes separately by gender.

\section{Procedures}

At the time of intake, all patients completed an informed consent that allowed their assessment data to be used for research purposes. As noted above, the MMPI-2 was completed in conjunction with an initial diagnostic evaluation. The OQ-45 was also administered at intake and at each subsequent session that the client attended. Because the focus of the study was on the amount of change over the course of therapy, the OQ-45 scores from the initial session and each subsequent session that the client attended were used in the analyses. Treatment included a mixture of individual cognitive-behavioral therapy (CBT) and interpersonal psychotherapy 
(IPT), with the predominant use of techniques such as an exploration of relationship issues, relaxation training, exposure with response prevention, challenging irrational beliefs, and behavioral activation. Therapists and supervisors generated individualized treatment plans. Although these treatments relied heavily on the use of empirically supported interventions, treatments were not manualized.

\section{Results}

The means and standard deviations for each of the MMPI-2 scales in the current study are presented in Table 1. The mean scores for the OQ-45 were 90.14 at initial testing and 65.23 after the final OQ-45 was administered. The number of sessions that patients attended varied widely, ranging from two to forty-nine $(\mathrm{M}=11.21, \mathrm{SD}=9.76$; Median $=8.00)$.

Table 1 MMPI descriptive statistics and results from HLM analyses

Table 1 MMPI descriptive statistics and results from HIM analyses

\begin{tabular}{|c|c|c|c|c|c|c|}
\hline & \multirow[b]{2}{*}{ Mean } & \multirow[b]{2}{*}{ SD } & \multicolumn{2}{|c|}{ Associaticn with hake $O Q$} & \multicolumn{2}{|c|}{$\begin{array}{l}\text { Association with Sy mpqoom } \\
\text { Change }\end{array}$} \\
\hline & & & $B$ & PV & $B$ & PV \\
\hline$L$ & 50 & $12 . \omega 2$ & $-.77^{44}$ & .16 & -04 &.$\infty$ \\
\hline$F$ & 71.08 & 18.97 & $.59 * 4$ & .22 & 03 & .04 \\
\hline$\kappa$ & 44.2 & 8. 73 & $-.77^{+}$ & 07 & -04 &.$\infty 0$ \\
\hline Hypochondriasis (Hs) & 65.22 & 14.02 & .23 & $\omega 2$ & $0 \mathrm{~s}^{*}$ & .13 \\
\hline Depressicn (D) & 73.27 & 13.75 & .28 & $\omega 2$ & $07^{4}$ & .09 \\
\hline Hysteria (Hy) & 65.92 & 15.01 & .30 & .03 & $07^{*}$ & .16 \\
\hline Psychopathic Deviance (Pd) & 65.84 & 13.83 & $.68^{24}$ & .15 & 01 &.$\infty 0$ \\
\hline Parancia (Pa) & 70.43 & 16.34 & $.44 * 4$ & .09 & 05 &.$\infty 0$ \\
\hline Psychastheria (Pt) & 73.49 & 14.64 & .16 &.$\infty$ & $.08^{+}$ & .21 \\
\hline Schizophreria (Sc) & 73.41 & 15.76 & $.55 *$ & .13 & .06 & .12 \\
\hline Hypomania (Ma) & 53.59 & 9.99 & .21 &.$\infty 0$ & .08 & .03 \\
\hline Ego Strength (Es) & 34.86 & 9.66 & $-.68^{+}$ &.$\infty 6$ & -03 &.$\infty$ \\
\hline Anxiety (Anx) & 72.95 & 10.37 & .56 &. $\cos$ & $.10^{+}$ & .15 \\
\hline Depressicn (Dep) & 70.4 & 11.45 & $.46^{+}$ & .05 & $.09^{+}$ & .11 \\
\hline Negative Treatmerk Inticators (Trt) & 66.45 & 14.34 & $.50^{*}$ & $\cos$ & 04 &.$\infty$ \\
\hline
\end{tabular}

** $\mathrm{P}<.01, * \mathrm{P}<.05,+\mathrm{P}<.10 . \mathrm{B}=$ unstandardized regression weights from Level 2 models. $\mathrm{PV}$ $=$ proportion of variance in intake symptom level or symptom change associated with each MMPI scale

Multilevel modeling (Fleeson 2007) with random effects was used to examine symptom-change. Conceptually, these analyses begin with a regression equation for each participant — with 
symptom-level regressed on a "session number" variable which begins at zero for the intake session and progresses by one for each subsequent session. For this within-person level of analysis, a participant's intercept reflects his or her symptom level at intake, and the slope represents the degree to which his or her symptom levels changed over sessions - a positive slope indicates increasing symptoms, and a negative slope indicates decreasing symptoms.

Initial analyses reveal that the average person's symptom level decreased across treatment sessions. This model included no predictors at the between-person level 2 (i.e., no MMPI-2 variables were used to explain differences between people). Results revealed that the average person had a symptom-change slope of $-1.91(\mathrm{t}=-3.405, \mathrm{P}=.002)$, indicating a significant degree of symptom decrease. Specifically, the average person's OQ-45 score decreased by nearly two points for each session attended.

As shown in Table 1, the next set of analyses revealed which MMPI-2 scales was related to: (a) symptom levels at intake, and (b) to symptom change. Conducted separately for each MMPI-2 scale, these models included an MMPI-2 scale and participants' number of sessions (as a control variable). Among the validity scales, L and F were statistically significant correlates of OQ-45 scores at intake. With respect to the $\mathrm{L}$ scale, relatively higher scores were associated with relatively lower OQ-45 scores during the initial session. Regarding the F scale, the association was positive, suggesting that relatively higher scores on $\mathrm{F}$ were related to relatively higher OQ45 scores at intake. Among the clinical scales, Pd, Pa (Paranoia), and Sc (Schizophrenia) were statistically significant correlates of intake OQ-45 scores. In each case, relatively higher scores on these clinical scales were associated with relatively higher OQ-45 scores during initial assessment. Of the four remaining MMPI-2 scales used, Trt was the only statistically significant predictor of OQ-45 level at intake.

Analyses further revealed that three clinical scales Hypochondriasis (Hs), Depression (D), and Hysteria (Hy) were statistically significant predictors of symptom change. For example, the positive unstandardized coefficient for $\mathrm{Hs}(\mathrm{B}=.08)$ indicates that elevated Hs levels are associated with larger within-person regression slopes. Considering the finding that the average person's symptoms decreased significantly across sessions (i.e., the average within-person regression slope was -1.9), the positive coefficient for Hs can be interpreted as showing that people with relatively high Hs scores manifested weaker symptom decreases than people with relatively low Hs scores. In general, Table 1 reveals that people with high scores on Hs, D, and Hy are less likely to show symptom reduction than are people with low scores. A specific example from the current study would be that for a patient whose Hs score is 1 SD above the mean for the sample (approximate T score of 75), the predicted regression slope per session (OQ score change) would be -1.11 instead of -1.91 as it is for average study participant. That is, for each unit change on the Hs, the change in slope would be .08 units (times the number of T-score points, in this case ten). Note that these scales (Hs, D, Hy) are distinct from the clinical scales that were associated with relatively higher OQ-45 scores at intake (i.e., Pd, Pa, Sc). Analyses 
including all MMPI-2 clinical scales in a single model revealed no unique effects, due to the overlap among the scales.

\section{Discussion}

The primary aim of the study was to assess the utility of the MMPI-2 in predicting symptom reduction as measured by the OQ-45 in an outpatient sample of psychotherapy patients who underwent a combination of CBT and IPT. The findings from this study suggest that whereas certain MMPI-2 scales (L, F, Pd, Pa, Sc, Trt) are most clearly associated with initial symptom levels, relatively higher scores on three other clinical scales, on average, predict less response to psychotherapy over time. In this case, relative elevations on Hs, D, Hy, portend less symptom reduction as measured by the OQ-45. Indeed, for a patient with a T-score on Hs that is $1 \mathrm{SD}$ above the sample mean, his or her predicted rate of symptom reduction per session would be almost half that of patients with a T-score near the sample mean. In essence, the utility of the MMPI-2 relative to psychotherapy outcome likely depends on the nature of the question being asked. For instance, when attempting to predict initial levels of distress, Pd might be more useful than some of the other MMPI-2 indices. However, a statistically significant association between $\mathrm{Pd}$ and symptom reduction over time is not evident based on the findings presented here, which is somewhat inconsistent with previous findings that suggest elevations on Pd were associated with poorer outcomes even after taking initial symptom levels into account (e.g., Forbes et al. 2002).

In addition to the significant associations reported for the aforementioned MMPI- 2 scales depending on the nature of the question being asked, we now turn our attention to what we did not find. For instance, other than a moderate correlation between a relatively higher score on Trt and relatively higher levels of distress based on initial OQ scores, there was little or no support for the associations between the MMPI-2 scales Es, Anx, and Dep, either at intake or over time based on these data.

Overall, our findings might suggest that when a clinician encounters a protocol with similar MMPI-2 profiles, particular clinical scales on average are potentially more useful at predicting outcome or symptom reduction as opposed to initial levels of distress. Moreover from a practical standpoint, clinicians might be well advised to take active steps in providing feedback to the patient (e.g., recommending a higher dosage of treatment) and possibly altering the treatment approach (e.g., being more attentive to debilitating levels of somatic symptoms and depression based on the Hs and D scores, adding a family therapy component to treatment). This might be especially true for patients with relatively higher scores on the Trt scale as well, which might be 
associated with higher initial levels of distress and a poorer response to psychotherapy. Indeed, Finn and Tonsager (1992) found that providing psychotherapy patients feedback regarding their MMPI-2 profiles after the initial assessment actually enhanced outcomes, including declines in symptomatic distress and feeling more hopeful about resolving their problems. Further, in a related investigation, Newman and Greenway (1997) studied the efficacy of providing feedback from MMPI-2 profiles and found that patients reported significant increases in self-esteem and significant decreases in symptomatic distress at a 2 week follow-up assessment compared to those that did not receive the feedback. Thus, although the findings from this study suggest that relatively higher scores on Hs, D, and Hy MMPI-2 portend worse outcome based on OQ scores over time, providing feedback about these data and other pertinent information to the patients early during the course of psychotherapy might actually alter or improve the results, given some of the aforementioned studies and the recent work of Lambert and colleagues (e.g., Lambert et al. 2001).

\section{Limitations}

Three limitations of these findings should be mentioned. First, the fact that not all patients in this sample received a DSM diagnosis might be viewed as a weakness. However, even those patients without a formal DSM diagnosis were on average, evidencing moderately elevated depressive symptoms as measured by the BDI and clinical levels of distress as measured by the OQ-45. The mean levels of depression and general distress were commensurate for those with or without a DSM diagnosis. Therefore, when considering all of the clinical data together, the patients in this sample are distressed and symptomatic to point where intervention was sought.

The second limitation of the study is the fact that the participants were served in a training clinic with clinicians who had varied prior experience. Further, patients received a variety of interventions within a general cognitive-behavioral and/or interpersonal paradigm, but were not treated in accord with a protocol-driven or manualized approach. As a result, it is difficult to estimate how the findings might generalize to more specific therapeutic approaches. Nevertheless, the broad range of both clients and treatments along with the number of therapists may, in fact, enhance the external validity of these findings in terms of generalization to psychotherapy practice as it typically occurs in outpatient settings. It remains of great interest in future research to determine how patient profiles on the MMPI-2 might interact with therapist variables (e.g., level of training, theoretical orientation). It may be that patients who did not respond well to the interventions provided in this setting would be responsive to services provided by more experienced clinicians or a different form of psychotherapy.

The third limitation is the fact that we were unable to re-calculate MMPI- 2 scores using the Revised Clinical Scales (Tellegen et al. 2003) due to the fact that the original raw data was unavailable. Using the RC scales would have been helpful in comparing the utility of the original MMPI-2 scales with their newer counterparts. And although our results provide a useful comparison point with previous investigations as well as helpful information for a considerable 
number of contemporary practitioners who still use the traditional scales, it is essential to make the evaluation of the RC scales commonplace in all future MMPI-2 studies of a similar nature.

In summary, the literature on psychotherapy outcome has clearly moved beyond the question of whether therapy is effective. In the present study, we examined the "effective for whom" question via an incorporation of personality predictors of treatment response. Our results suggest that the MMPI-2 can play a useful role in assessing which patients might have a harder time making improvements during the course of psychotherapy and not simply who might be at risk for higher levels of distress at intake. Ideally, clinicians who opt to use the MMPI-2 as an assessment instrument can potentially expand the utility of the device by using patients' MMPI-2 data to assist in making evidence-based decisions about which patients will be well-served in their care, which will require a revision to the treatment such as an increased dosage, and which may benefit from an alternative treatment.

\section{References}

American Psychiatric Association. (2000). Diagnostic and statistical manual of mental disorders - fourth edition - text revision. Washington, DC: American Psychiatric Press.

Barron, F. (1953). An ego-strength scale which predicts response to psychotherapy. Journal of Consulting Psychology, 17, 327-333. doi:10.1037/h0061962.

Butcher, J. N., Graham, J. R., Williams, C. L., \& Ben-Porath, Y. S. (1990). Development and use of the MMPI-2 content scales. Minneapolis: University of Minnesota Press.

Butcher, J. N., Graham, J. R., Ben-Porath, Y. S., Tellegen, A., Dahlstrom, W. G., \& Kaemmer, B. (2001). Minnesota Multiphasic Personality Inventory-2 (MMPI-2): Manual for administration and scoring: Revised edition. Minneapolis: University of Minnesota Press.

Chisholm, S. M., Crowther, J. H., \& Ben-Porath, Y. S. (1997). Selected MMPI-2 Scales' ability to predict premature termination and outcome from psychotherapy. Journal of Personality Assessment, 69, 127-144. doi:10.1207/s15327752jpa6901_7.

Clark, M. E. (1996). MMPI-2 negative treatment indicators content and content component scales: Clinical correlates and outcome prediction for men with chronic pain. Psychological Assessment, 8, 32-38. doi:10.1037/1040-3590.8.1.32.

Craig, R. J., \& Olson, R. E. (2004). Predicting methadone maintenance treatment outcomes using the addiction severity index and the MMPI-2 content scales (negative treatment indicators and 
cynicism scales). The American Journal of Drug and Alcohol Abuse, 30, 823-839. doi:10.1081/ADA-200037548.

Eysenck, H. J. (1952). The effects of psychotherapy: An evaluation. Journal of Consulting Psychology, 16, 319-324. doi:10.1037/h0063633.

Finn, S. E., \& Tonsager, M. E. (1992). Therapeutic effects of providing MMPI-2 test feedback to college students awaiting therapy. Psychological Assessment, 4, 278-287. doi:10.1037/10403590.4.3.278.

Fleeson, W. (2007). Studying personality processes: Explaining change in between-persons longitudinal and within-person multilevel models. In R. Robins, C. Fraley, \& R. Krueger (Eds.), Handbook of research methods in personality psychology (pp. 523-542). New York: Guilford Press.

Forbes, D., Creamer, M., Allen, N., Elliot, P., McHugh, T., Debenham, P., et al. (2002). The MMPI-2 as a predictor of symptom change following treatment for posttraumatic stress disorder. Journal of Personality Assessment, 79, 321-336. doi:10.1207/S15327752JPA7902_13.

Garfield, S. L. (1994). Research on client variables in psychotherapy. In A. E. Bergin \& S. L. Garfield (Eds.), Handbook of psychotherapy and behavior change (4th ed., pp. 190-228). New York: Wiley.

Getter, H., \& Sundland, D. M. (1962). The Barron ego-strength scale and psychotherapy outcome. Journal of Consulting Psychology, 26, 195. doi:10.1037/h0047960.

Gilmore, J. D., Lash, S. J., Foster, M. A., \& Blosser, S. L. (2001). Adherence to substance abuse treatment: Clinical utility of two MMPI-2 scales. Journal of Personality Assessment, 77, 524540. doi:10.1207/S15327752JPA7703_11.

Graham, J. R. (2005). MMPI-2 assessing personality and psychopathology (4th ed.). New York: Oxford University Press.

Hathaway, S. R., \& McKinley, J. C. (1983). The Minnesota multiphasic personality inventory manual. New York: Psychological Corporation.

Helmes, E., \& Reddon, J. R. (1993). A perspective on developments in assessing psychopathology: A critical review of the MMPI and MMPI-2. Psychological Bulletin, 113, 453-471. doi:10.1037/0033-2909.113.3.453.

Hill, C. E., \& Lambert, M. J. (2003). Methodological issues in studying psychotherapy processes and outcomes. In M. J. Lambert (Ed.), Bergin and Garfield's handbook of psychotherapy and behavior change (5th ed., pp. 108-109). New York: Wiley.

Hilsenroth, M. J., Handler, L., Toman, K. M., \& Padawer, J. R. (1995). Rorschach and MMPI-2 indices of early psychotherapy termination. Journal of Consulting and Clinical Psychology, 63, 956-965. doi:10.1037/0022-006X.63.6.956.

Lambert, M. J., Burlingame, G. M., Umphress, V., Hansen, N. B., Vermeersch, D. A., Clouse, G. C., et al. (1996a). The reliability and validity of the Outcome Questionnaire. Clinical Psychology 
\& Psychotherapy, 3(4), 249-258. doi:10.1002/(SICI)1099-0879(199612)3:4<;249::AIDCPP106>;3.0.CO;2-S.

Lambert, M. J., Hansen, N. B., Umphress, V., Lunnen, K., Okiishi, J., Burlingame, G. M., et al. (1996b). Administration and scoring manual for the OQ45.2. Stevenson, MD: American Professional Credentialing Services, LLC.

Lambert, M. J., Hansen, N. B., \& Finch, A. E. (2001). Patient-focused research: Using patient outcome data to enhance treatment effects. Journal of Consulting and Clinical Psychology, 69, 159-172. doi:10.1037/0022-006X.69.2.159.

Lambert, M. J., Bergin, A. E., \& Garfield, S. L. (2003). Introduction and historical overview. In M. J. Lambert (Ed.), Bergin and Garfield's handbook of psychotherapy and behavior change (5th ed., pp. 3-15). New York: Wiley.

Lambert, M. J., Morton, J. J., Hatfield, D., Harmon, C., Hamilton, S., Shimokawa, K., et al. (2004). Administration and scoring manual for the OQ45.2. Stevenson, MD: American Professional Credentialing Services, LLC.

Lima, E. N., Stanley, S., Kaboski, B., Reitzel, L. R., Richey, A., Castro, Y., et al. (2005). The incremental validity of the MMPI-2: When does therapist access not enhance treatment outcome? Psychological Assessment, 17, 462-468. doi:10.1037/1040-3590.17.4.462.

Luborsky, L., Mintz, J., Auerbach, A., Christoph, P., Bachrach, H., \& Todd, T. (1980). Predicting the outcome of psychotherapy: Findings of the Penn Psychotherapy Project. Archives of General Psychiatry, 37, 471-481.

Masters, K. S., Shearer, D. S., Ogles, B. M., \& Schleusener, R. L. (2003). Pre-surgical MMPI-2 cluster profiles predict one-year low-back surgery outcomes. Journal of Clinical Psychology in Medical Settings, 10, 279-288. doi:10.1023/A:1026301420769.

Minnix, J. A., Reitzel, L. R., Repper, K. A., Burns, A. B., Williams, F., Lima, E. N., et al. (2005). Total number of MMPI-2 clinical scale elevations predicts premature termination after controlling for intake symptom severity and personality disorder diagnosis. Personality and Individual Differences, 38, 1745-1755. doi:10.1016/j.paid.2004.11.006.

Newman, M. L., \& Greenway, P. (1997). Therapeutic effects of providing MMPI-2 test feedback to clients at a university counseling service: A collaborative approach. Psychological Assessment, 9, 122-131. doi:10.1037/1040-3590.9.2.122.

Shepherd, K. (1997). Prediction of treatment outcome using the MMPI-2. Unpublished doctoral dissertation. Kent: Kent State University.

Smith, M. L., Glass, G. V., \& Miller, T. L. (1980). Benefits of psychotherapy. Baltimore: John Hopkins University Press.

Tellegen, A., Ben-Porath, Y. S., McNulty, J. L., Arbisi, P. A., Graham, J. R., \& Kaemmer, B. (2003). MMPI-2 Restructured Clinical (RC) scales: Development, validation, and interpretation. Minneapolis: University of Minnesota Press. 
Vendrig, A. A., Derksen, J. J. L., \& de Mey, H. R. (2000). MMPI-2 personality psychopathology five (PSY-5) and prediction of treatment outcome for patients with chronic back pain. Journal of Personality Assessment, 74, 423-438. doi:10.1207/S15327752JPA7403_6.

Vermeersch, D., Lambert, M. J., \& Burlingame, G. M. (2000). Outcome Questionnaire: Item sensitivity to change. Journal of Personality Assessment, 74, 242-261.

doi:10.1207/S15327752JPA7402_6. 